\title{
IMPROVING FAKE PRODUCT DETECTION USING AI-BASED TECHNOLOGY
}

\author{
Eduard Daoud, Dang Vu, Hung Nguyen and Martin Gaedke \\ Technische Universität Chemnitz, Germany
}

\begin{abstract}
ResearchAndMarkets wrote in their report on May 15, 2018, that up to 1.2 Trillion USD in 2017 of products are counterfeited goods. The report estimated this damage globally at 1.82 Trillion USD in 2020 (RESEARCH AND MARKETS, 2018). This paper does not consider copyrights or digital piracy, counterfeiting, fraudulent documents but rather investigates the prevention of counterfeiting on a technological basis. The presence of counterfeit products on the European market is on the increase, therefore the intervention of inspection bodies and authorities alone is not sufficient, consumers can make their contribution and support this process. In this paper, we research the possibility to reduce counterfeit products using machine learning-based technology. Image and text recognition and classification based on machine learning have the potential to be a key technology in the fight against counterfeiting. The automatic image and text recognition and the classification of product information enable end customers to detect counterfeits precisely and quickly by checking them against trained models. The goal of this paper is to create an easy to use applications in which the end-user identifies the counterfeit product and contribute to the fight against product piracy.
\end{abstract}

\section{KEYWORDS}

Anti-Counterfeiting, Machine Learning, Deep Learning, Image Recognition, Object Detection

\section{INTRODUCTION AND CURRENT PROBLEM}

Detection of counterfeit products is in certain cases a challenge for the consumers and can sometimes be dangerous when it comes to medical products or toys for children, for example. ResearchAndMarkets wrote in their report on May 15, 2018, that up to 1.2 Trillion USD in 2017 of products are counterfeited goods. The report estimated this damage globally at 1.82 Trillion USD in 2020 (RESEARCH AND MARKETS, 2018).

Especially The presence of counterfeit products on the European market is on the increase (OECD/EUIPO, 2016), therefore the intervention of inspection bodies and authorities alone is not sufficient. Since its launch in 2003, the EU's Rapid Alert System has been providing EU member States with a network and communication tools to publicize counterfeit products. The system stabilizes at regular intervals, about 50 alerts are published each week on the European Commission's website, with slightly more than 2,000 alerts released each year. (Directorate-General for Justice and Consumers (European Commission), 2018). The number of counterfeits reported products is extremely low in relation to the number of counterfeit products imported into the EU. OECD wrote in their report 2016 that up to $5 \%$ of imports are counterfeited goods. The report estimated this damage at EUR 85 billion (OECD/EUIPO, 2016). A major problem of such governmental instruments is that the end-consumers is not involved, if at all, in the detection process of counterfeiting.

In the next section, we will highlight the subject of counterfeit domains and focus on the area where the use of IT technology can make a positive contribution. After introducing the related works, we will outline the solution concept and technical architecture, then we will focus on the implementation and evaluation of such solutions and their challenges. Finally, we will review the results of our work and consider the outlook for the future. 


\section{RELATED WORKS}

In general, anti-counterfeiting technologies should be easily applied and used by end consumer first but challenging to imitate and ensure that businesses are safeguarded financially and in terms of reputation. Based on $(\mathrm{Li}, 2013)$ these technologies usually have four main features:

- difficult to duplicate or forge,

- $\quad$ easily identifiable visually without the need of special equipment,

- hard to re-label or reuse, and

- $\quad$ easily noticeable when tampered with.

From a product standpoint, there are three common categories for anti-counterfeiting: overt, covert, and track and trace, shown in Figure 1. Overt technologies focused on the packaging of the products. Color-shifting inks, watermarks and holograms are some of the technologies that can be used in this category. End consumers need to be briefed in advance so that they interpret these technologies correctly to verify the fake products. Covert technologies like ultraviolet (electromagnetic radiation) and bi-fluorescent are also applied to the product itself but are not identifiable without special equipment. Digital watermarks, hidden printed messages and pen-reactive ink. are also part of the covert technologies.

The final category is track and trace. Radio Frequency Identification (RFID) tags, Electronic Product Codes (EPCs) and barcodes are the main technologies in this category. The effect of holistic tracking and tracing contributes that the reduction of counterfeits is possible. Consumers and retailers scan the code already implemented by suppliers and manufacturers to verify the authenticity of the product or to trace the overall supply chain process.

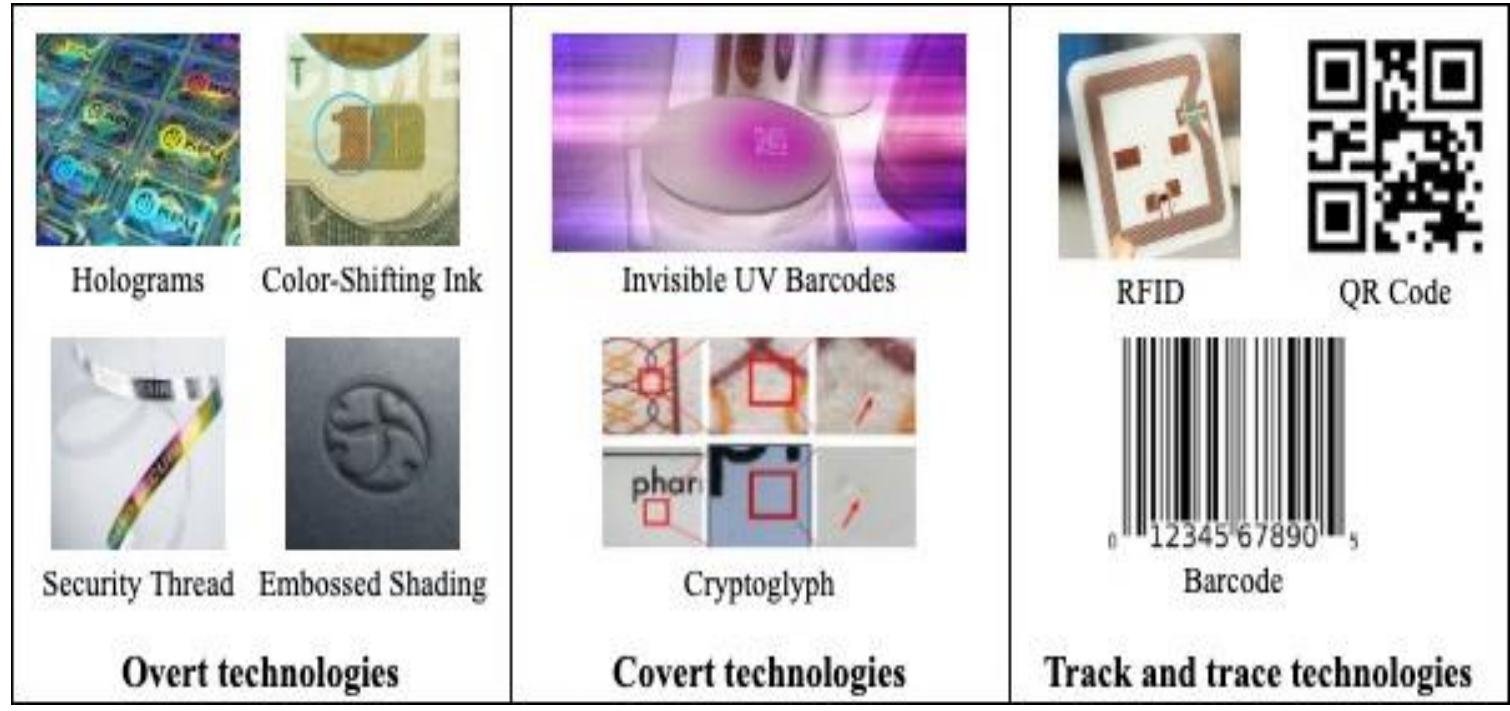

Figure 1. Anti-counterfeiting technologies 
There are other approaches based on improved communication between companies and organizations with the interest to reduce counterfeiting on the market - an example is React. React is a not-for-profit organization providing a market, online and customs enforcement professional services (React, 2020). Professional services approaches have a big advantage concerning accuracy but still working with the manual process and need manpower. All three technologies mentioned in Figure 1. Anti-counterfeiting technologies have disadvantages and limitations. In previous researches, we have addressed the subject in detail but with the use of blockchain technologies (Daoud \& Gaedke, 2019) and we found also a lot of limitations. Counterfeiters are becoming more and more professional and sophisticated. They are always developing approaches to better package counterfeit products and bring them to the market undetected (Shields \& Deshmukh, 2020). Consequently, overt and covert technologies can be easily imitated. It is difficult for the average end-consumer can distinguish between a convincing imitation and the real product. Besides, covert technologies are required special devices to identify counterfeited products so that customers are neither able to detect nor verify their products. The track and trace technologies with its encoding and security feature can be used in combination to improve this situation but it leads to another major issue: overhead cost. As a consequence, the price of the product has risen further, which in turn leads the end-user to look for counterfeit products in certain cases. (Li, 2013).

Based on that, this paper proposes a low-cost and user-friendly solution relies on machine learning-based technology which enables end-consumers to identify and verify products without any special equipment. By using image and text recognition, this approach aims to improve fake product detection. It can also combine with over, covert and/or Track and Trace technologies to help combat counterfeiting more efficient and effective. In the next section, we will introduce the concept behind the solution and the technical architecture after that we will go deeper in the implementation stage.

\section{CONCEPT AND TECHNICAL ARCHITECTURE}

According to the report of (Statista, 2019), the current number of mobile phone users in the world is 4.78 billion, of which 3.5 billion are smartphone users. Today, with cheap price, users can easily own a smartphone with a built-in digital camera and internet access. Based on that, the proposed solution will allow the end-consumers to use their phones as equipment to detect fake products.

For detection, the end-consumer takes pictures of a product packaging, which contains product text information, logos and maybe certification marks/logos. These pictures will be sent in a request to the server for processing and verifying. Afterwards, the detection result will be returned to the end-consumer to make a further decision. In the case of fake product detection, the end-consumer has an ability to report this counterfeit product to the government system, such as the Safety Gate - EU's Rapid Alert System. An overall concept of this solution is shown in Figure 2.

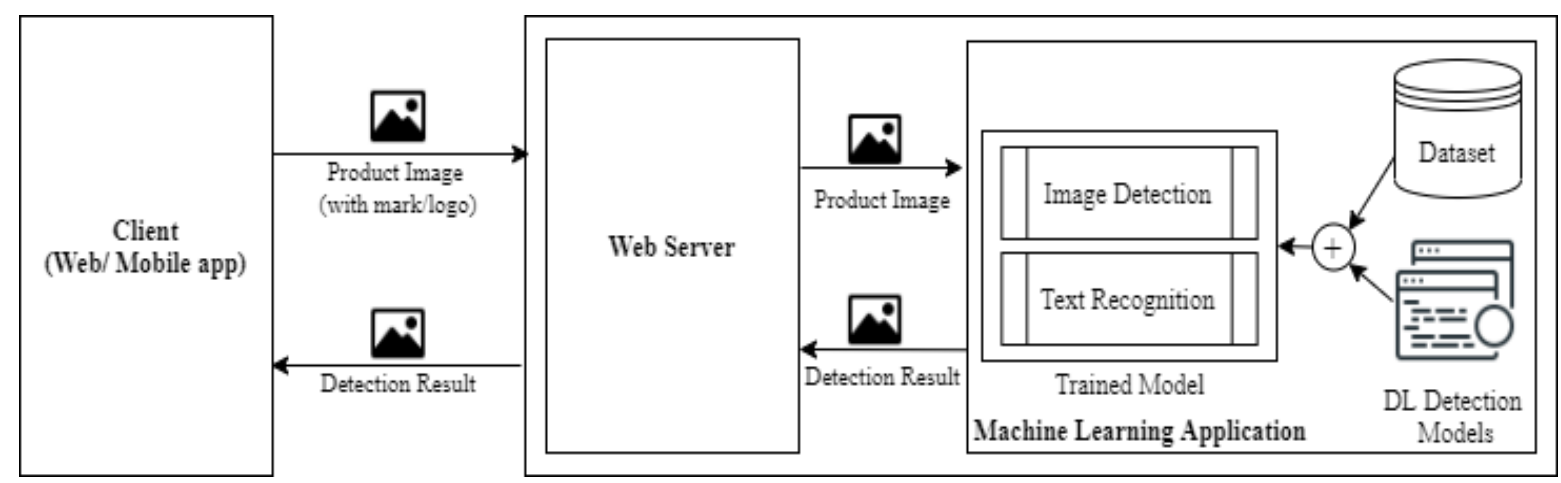

Figure 2. Overall Concept 
In the server-side, there are two main components: a web server and a machine learning application. The web server will be acted as the middle layer. It receives requests (pictures and metadata) from the end-users (the client-side) and forwards this request information to the machine learning application for detection. After processing, the web server receives the detection result and sent the response back to the client. In addition, the server also performs several operations such as storing detection results, data statistic or allowing users to report counterfeit products.

The machine learning application is the main contribution of this paper. An architecture of this application has three main parts: the dataset, detection models, and trained model. In order to have an efficient machine learning application, we need first a dataset represents the related entities and their values. There are two types of dataset: training dataset and test dataset. In our application, dataset is a collection of valid logo or/and mark.

Next, we need the detection models- the functions/algorithms which perform certain operations on the given input (product images with mark/logo) and procedure the suitable output (detection result). More specifically, we apply the pre-trained Deep Learning (DL) detection models, e.g. SSD (Liu, et al., 2016) and Faster R-CNN (Ren, et al., 2016), which will be discussed in detail in section 4. To create the trained model, we do a transfer learning by training the pre-trained detection models with our input dataset. Our trained model contains two key components: image detection and text recognition, which aims to verify the validity of the mark/logo. Image detection and text recognition can be applied in a wide range of application, from optical character recognition - OCR (machine translation, (extracted) text-to-speech), face detection, face recognition, self-driving cars, to medical imaging, and robotics (Vahab, et al., 2019).

To challenge the concept and the proposed solution is the first machine learning application particularly concentrating on detecting fake product, we implement the solution in the next section and training the model with the certification body logo and the mark of TÜV SÜD AG. There are many reasons to assume, and based on the (OECD/EUIPO, 2016), that many counterfeiters use the certification agency logo to gain better access to the EU market.

\section{IMPLEMENTATION}

Figure 3 shows a detailed implementation of the approached machine learning detection solution. The implementation consists of two steps: training models step and detecting logo step. In the first step, the training model component depicts a way we prepare dataset and select the Deep Learning pre-trained detection model. For demonstration, we collect 2000 certification marks and logos from the websites (web crawler) of the Testing, Inspection and Certification (TIC) members, e.g. TÜV SÜD AG, Dekra and Bureau Veritas. To make our dataset more diverse and avoid overfitting, we use another technique also to enrich our dataset by extracting the certification marks and logos from video frames.

The more data we have, the higher the accuracy of our model can perform. After collecting dataset, we label our raw data by using annotation tools (i.e., LabelImg) and divide them into a training set (80\%) and test set $(20 \%)$. The collecting-labelling is not a one-time process. We operate it in an iterative fashion so that the machine learning model could gain the ability to generalize and return more precisely outcome. 


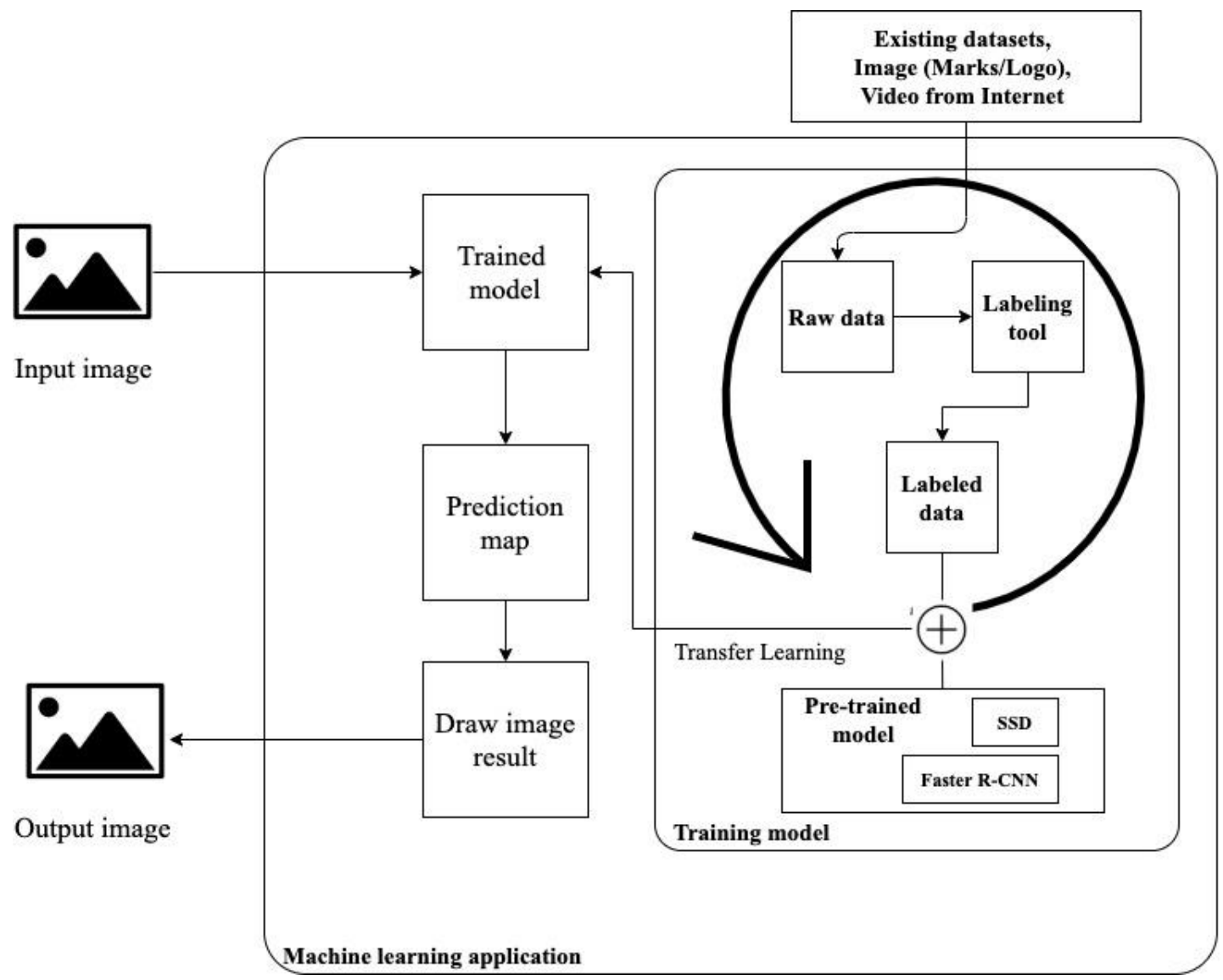

Figure 3. Implementation of machine learning detection application

Instead of investing time and effort for building a trained model from scratch, we use transfer learning- an optimization that allows rapid progress and boots performance when modelling our detection task. Two pre-trained models are used in our transfer learning are SSD300 - Regression/Classification based family- and Faster R-CNN Resnet101 - Region-based family-. Based on (Zhao, et al., 2019), Faster RCNN archive high accuracy (mean Average precision of $83.8 \%$ by testing on VOC 07 Test set) with low training speed.

Because of efficiently detecting small image resolution with detailed features, it is used to verify certification marks. On the other hand, SSD can run fast and smooth (test time of 0.02 second/image and rate of 45 frames/second, tested on VOC 07 Test set) on identifying large objects, such as logo, with high precision.

The second step focuses on handling request from the webserver (product images with mark/logo) and returns detection result. When an image is passed through our trained model, a prediction map will be returned to classify and localize the certification mark/logo we need to detect. To perform visualizations for end-user, the prediction bounding box and the class label for each certificate mark are drawn on the uploaded, shown in Figure 4 in the next section. For demonstration, we construct a web application by using Flask - a lightweight Python web application framework and Tensorflow - an end-to-end open-source library to deploy our machine learning application. In the following section, we discuss the challenges of our approach bevor we headline the summary and an outlook on our work.

\section{EVALUATION AND CHALLENGES}

The approached solution has several improvements for the current anti-counterfeiting technologies. Firstly, it can be verified by average end-users, which can add a new protection layer to combat counterfeiting products. In comparison to the track and trace technologies mentioned in section 2, our solution provides a low-cost implementation, which is appropriate when the market is scaling up. 
In addition, unlike overt technology, end-consumer does not need any special device to use our solution they only need a mobile application and internet access to verify the genuineness of certification logo/marks. The detection result from our demonstration reveals the potential of machine learning-based technology to fight against counterfeiting. Our solution archives $97 \%$ precision at 3.1 seconds/certificate mark, on 400 tested data. As the result in Figure 4, the sophisticated forged marks can be detected, e.g. minor change in color, missing or incorrect text.

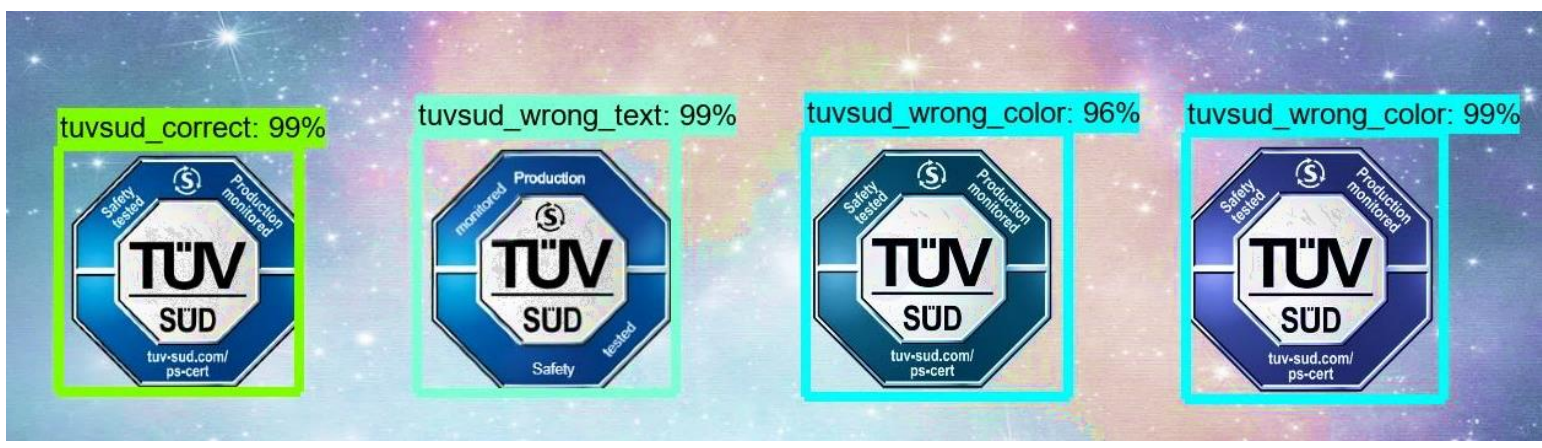

Figure 4. Tested results on TÜV SÜD AG certification mark

Similar to any other anti-counterfeiting technologies, issues and challenges arise for our machine learning-based approach. One of the challenges is the limitation of data for the training process. The amount of needed data to combat counterfeiting products is huge, but the existing data sources are limited. Furthermore, crawling data from the Internet and manually annotating consumes a lot of time. The more data we have, the more accuracy we can provide. Last but not least, 3.1 seconds for single certification mark detection is not efficient in real-time application. Hence, there is still room for improvement, e.g. considering other faster algorithms like YOLO (Redmon \& Farhadi, 2019) but with the trade-off of accuracy. After highlighting the challenges, we headline a summary and an outlook on our work.

\section{CONCLUSION AND OUTLOOK}

This paper presents a new approach as an anti-counterfeiting machine learning-based solution to detect fake product. We evaluate the solution by using machine learning and found a lot of limitations. The machine learning-based approach used in core deep learning and neural network technologies. The conclusions we can define from the new approach are that the implementation of the system should be deeper researched, from the point of view of collecting more training data and annotation/labelling service. The main focus is on which implementations have a positive impact on anti-counterfeiting of products and the adoption of machine learning-based detection depends on how the consumer can easily and simply interact with the system. By using image recognition, this approach could improve fake product detection. It can also combine with over, covert and/or track and trace technologies to help combat counterfeiting more efficient and effective.

In future work, we plan from one hand to explore and research more about using faster machine learning algorithms to classify marks and logos and detect text with the help of OCR. from the other hand, we need to extend our web crawler to have the possibility to gather more web information, especially from the eCommerce world to find fake products with help of detecting logo, marks and text. This would combine three state-of-the-art technologies, machine learning, Text recognition and web searching in one application. That will bring great convenience and a better experience for users. However, we trust that using machine learningbased technology will change the role and empower the consumer to drive the market for more transparency and safety. 


\section{REFERENCES}

Daoud, E. \& Gaedke, M., 2019. Decentralizing Products Certificates Using Blockchain. Cagliari, Italy, 18th International Conference on WWW/Internet, p. 8.

Directorate-General for Justice and Consumers (European Commission), 2018. Results of the EU rapid alert system for dangerous non-food products, Brussels: European Commission.

Li, L., 2013. Technology designed to combat fakes in the global supply chain. Business Horizons, 56(2), pp. 167-177.

Liu, W. et al., 2016. SSD: Single Shot MultiBox Detector. s.l., arXiv preprint, arXiv:1512.02325.

OECD/EUIPO, 2016. Trade in Counterfeit and Pirated Goods: Mapping the Economic Impact. Paris: OECD Publishing.

React, 2020. [Online] Available at: https://www.react.org/ [Accessed 0702 2020].

Redmon, J. \& Farhadi, A., 2019. Yolov3: An incremental improvement. s.l., arXiv preprint arXiv:1804.02767.

Ren, S., He, K., Girshick, R. \& Sun, J., 2016. Faster R-CNN: Towards Real-Time Object Detection with Region Proposal Networks. s.l., In Advances in neural information processing systems (pp. 91-99)..

RESEARCH AND MARKETS, 2018. Global Brand Counterfeiting Report, s.l.: RESEARCH AND MARKERTS.

Shields, D. \& Deshmukh, R., 2020. Anti-Counterfeit Packaging Market - Food And Pharmaceuticals by Authentication Packaging Technology., 97220 United States: Global Opportunity Analysis and Industry Forecast.

Statista, 2019. Number of smartphone users worldwide from 2016 to 2021, s.l.: Statista.

Vahab, A., Naik, M. S., Raikar, P. G. \& R, P. S., 2019. Applications of Object Detection System. International Research Journal of Engineering and Technology (IRJET), 06(04), pp. 4186-4192.

Zhao, Z., Zheng, P., Xu, S. \& Wu, X., 2019. Object detection with deep learning: A review.. EEE transactions on neural networks and learning systems, 30(11), pp. 3212-3232. 